Supporting Information

\title{
Crystal structure of the anthrax drug target, Bacillus anthracis dihydrofolate reductase
}

Brad C. Bennett, Hai Xu, Richard F. Simmerman, Richard E. Lee and Chris G. Dealwis

Contents

Supporting Table $1 \quad$ page $\mathrm{S} 2$

Supporting Figure $1 \quad$ page S3

Supporting Figure 2 pages S4-S5

Supporting Figure 3 page S6

Supporting References page S7 
Supporting Table 1: Reported $\mathrm{IC}_{50}$ values (in $\mu \mathrm{M}$ ) for DHFR inhibitors

\begin{tabular}{l|c|c|c|c|c} 
& MTX & TMP & MANIC & Iclaprim $^{\text {e }}$ & AR-709 \\
\hline baDHFR $^{\mathrm{a}, \mathrm{b}}$ & 0.015 & 77.2 & 104.2 & n.d. & n.d. \\
\hline hsDHFR $^{\mathrm{c}}$ & 0.024 & $>1000$ & n.d. & $>300$ & $>300$ \\
\hline saDHFR $^{\mathrm{d}}$ & n.d. & 45 & n.d. & 0.007 & 0.9 \\
\hline spDHFR $^{\mathrm{d}}$ & n.d. & 42 & n.d. & 0.008 & 0.09
\end{tabular}

${ }^{\mathrm{a}}$ The $\mathrm{IC}_{50}$ values for MTX and MANIC are reported from the present work.

${ }^{\mathrm{b}}$ The $\mathrm{IC}_{50}$ value for TMP against baDHFR was reported in ${ }^{1}$.

${ }^{\mathrm{c}}$ The $\mathrm{IC}_{50}$ values for MTX and TMP against hsDHFR were reported in ${ }^{1}$, and the $\mathrm{IC}_{50}$ value for AR-709 against hsDHFR was reported in ${ }^{2}$.

${ }^{\mathrm{d}}$ The $\mathrm{IC}_{50}$ values of AR-709 against hsDHFR, against DHFR from a TMP resistant Staphylococcus aureus mutant (saDHFR; F98Y) and against DHFR from a TMP resistant Streptococcus pneumoniae mutant (spDHFR; I100L) were reported in ${ }^{2}$.

${ }^{\mathrm{e}}$ The $\mathrm{IC}_{50}$ values shown for Iclaprim (formerly AR-100) were reported in ${ }^{3}$ and ${ }^{4}$.

n.d.: not determined 


\section{Supporting Figures}

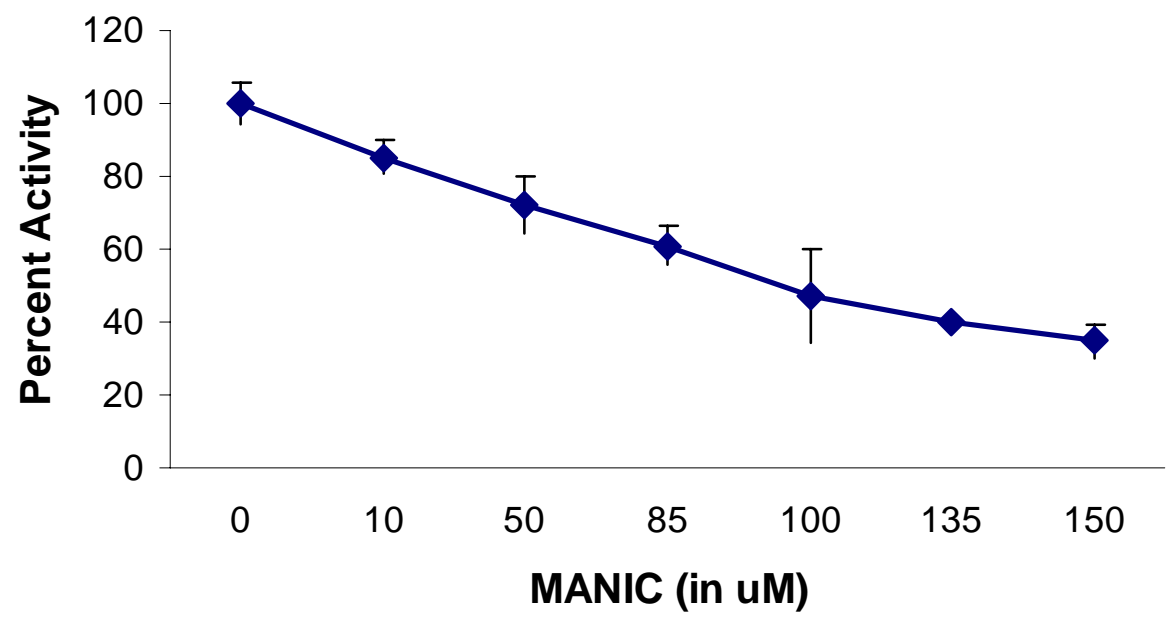

Supporting Figure 1. MANIC shows inhibitory activity against baDHFR. DHFR activity was measured at $340 \mathrm{~nm}$ (to monitor the oxidation of NADPH to $\mathrm{NADP}^{+}$) over the course of $90 \mathrm{~s}$ in the absence and in the presence of different concentrations of MANIC. We determined that the $\mathrm{IC}_{50}$ of MANIC for baDHFR is $104 \mu \mathrm{M}$. The concentration of enzyme and NADPH were $50 \mathrm{nM}$ and $12.5 \mu \mathrm{M}$, respectively. The concentration of the DHF substrate was held constant at $125 \mu \mathrm{M}$, which is assumed to be saturating. However, in a separate set of experiments, MANIC showed competitive inhibition of baDHFR at lower $(2.5,5,10,12.5$ and $15 \mu \mathrm{M})$ substrate concentrations. 


\section{a}
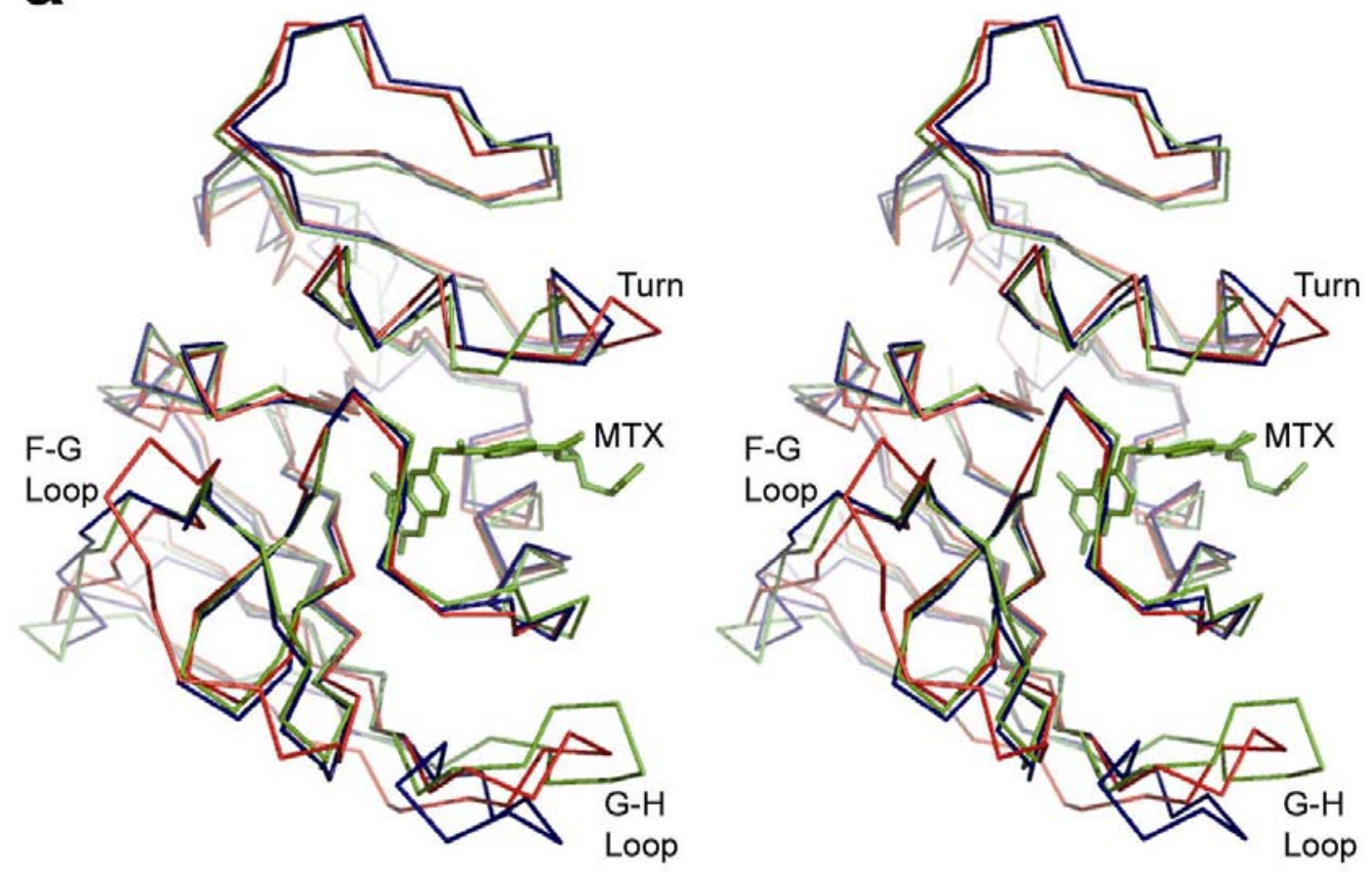

b
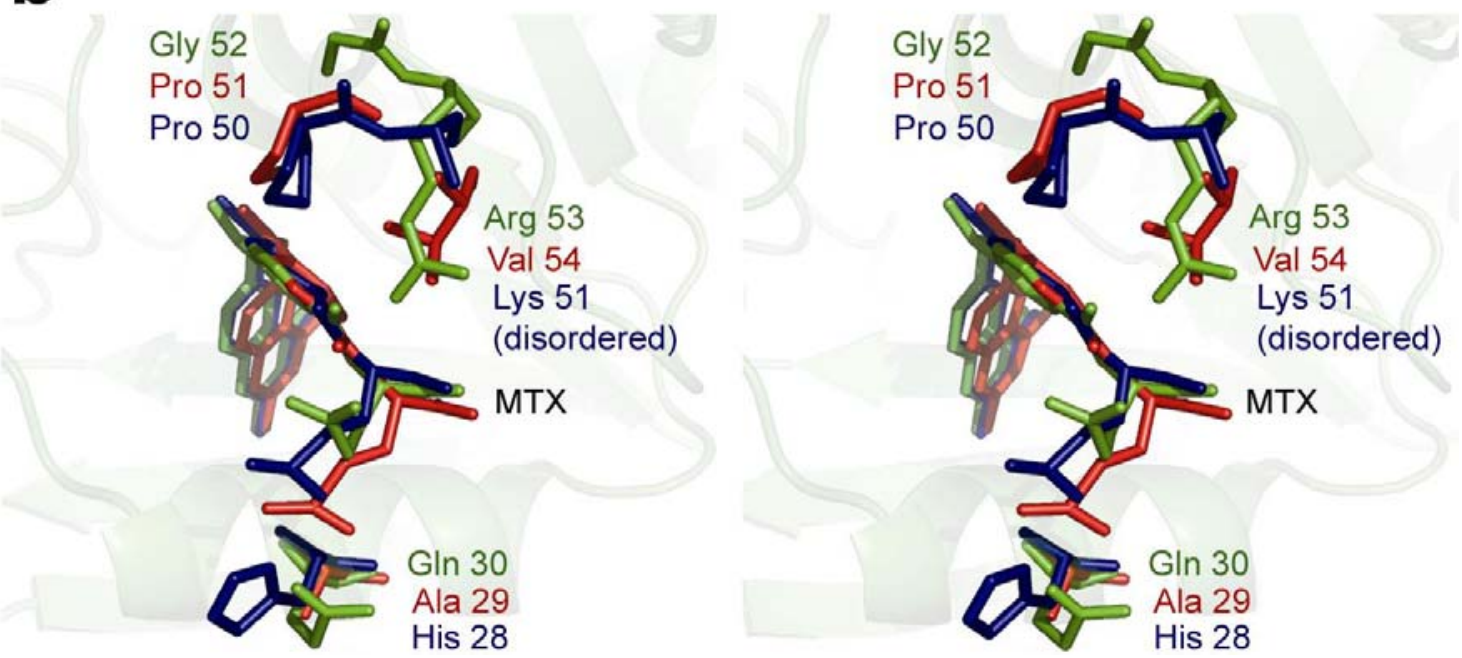

Supporting Figure 2. Structural differences between pathogenic prokaryotic DHFRs. (a) Stereo view of the superposition of Lactobacillus casei (lcDHFR; dark blue) and Mycobacterium tuberculosis (mtDHFR; red) DHFRs onto baDHFR (green), all shown as $\mathrm{C} \alpha$ traces. Regions of significant contiguous divergence between $\mathrm{C} \alpha$ atoms are labeled. The MTX molecule shown (green) is from the baDHFR structure. The superposition was performed using $\operatorname{Coot}^{5}$. (b) An up close stereo view of the active sites of the superposed DHFR molecules, with residue differences between the three structures shown (coloring as in panel A). Contacts to the pteridine ring of MTX are conserved but interactions formed 
with the $p$-ABA-Glu tail differ between the structures. Note the Gly (baDHFR) $\rightarrow$ Pro (lcDHFR and mtDHFR) substitution at position 52 of the baDHFR sequence. This corresponds to position 61 in hsDHFR and is also a Pro. 

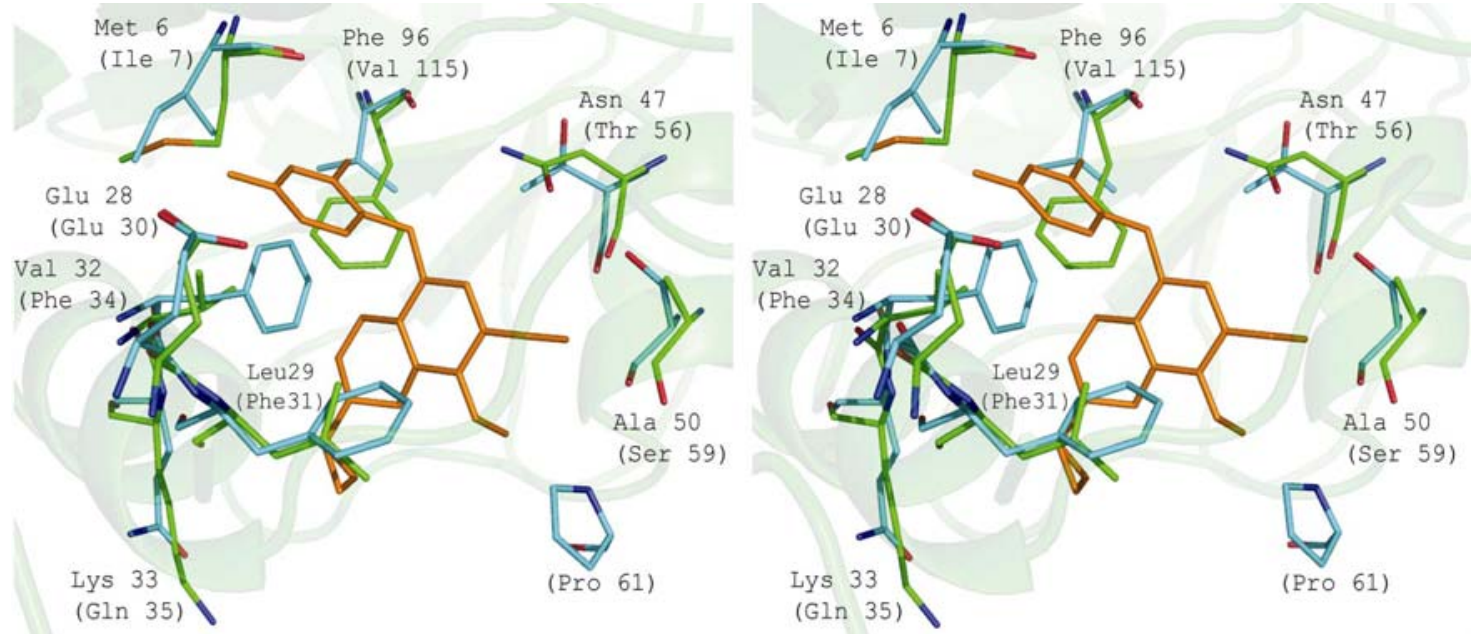

Supporting Figure 3. The diaminopyrimidine iclaprim may bind preferentially to baDHFR compared to hsDHFR. Stereo view of iclaprim (orange) modeled into the baDHFR active site. Iclaprim was docked into the baDHFR (carbon: green, oxygen: red, nitrogen: blue, sulfur: orange) active site and then the complex was energy minimized. The resultant conformation of iclaprim reveals that it does not clash with baDHFR but forms very close contacts with Phe 34, Phe 31 and Pro 61 of hsDHFR (carbon: cyan, oxygen: red, nitrogen: blue, sulfur: orange). 


\section{Supporting References}

(1) Barrow, E. W.; Bourne, P. C.; Barrow, W. W. Functional cloning of Bacillus anthracis dihydrofolate reductase and confirmation of natural resistance to trimethoprim. Antimicrob Agents Chemother 2004, 48,4643-4649.

(2) Mukhija, S.; Bandera, M.; Parisi, S.; Rigo, S.; Lieb, S. et al. AR-709- An Investigational diaminopyrimidine: Inhibition, Binding and Mode of Action. ICAAC - Interscience Conference on Antimicrobial Agents in Chemotherapy, 2006.

(3) Hartman, P. G.; Kompis, I.; Jaeger, J.; Mukhija, S.; Islam, K. AR-100, a novel diaminopyrimidine, can overcome TMP-resistance in staphylococci and streptococci. ICAAC: Interscience Conference on Antimicrobial Agents and Chemotherapy; American Society of Microbiology: Washington, D.C., 2002.

(4) Schneider, P.; Hawser, S.; Islam, K. Iclaprim, a novel diaminopyrimidine with potent activity on trimethoprim sensitive and resistant bacteria. Bioorg Med Chem Lett 2003, 13,4217-4221.

(5) Emsley, P.; Cowtan, K. Coot: model-building tools for molecular graphics. Acta Crystallogr D Biol Crystallogr 2004, 60,2126-2132. 